DOI: https://doi.org/10.24297/jam.v21i.9101

\title{
Distributions generated by the boundary values of functions in Privalov spaces
}

\author{
Mejdin Saliji ${ }^{1}$, Bedrije Bedzeti ${ }^{2}$, Vesna Manova Erakovikj ${ }^{3}$ \\ ${ }^{1}$ Faculty of Education, Ss. Ukshin Hoti, Prizren, Kosovo \\ ${ }^{2}$ Faculty of Mathematics and Natural Sciences, State University of Tetovo, Tetovo, Republic of North \\ Macedonia. \\ ${ }^{3}$ Faculty of Mathematics and Natural Sciences, Ss. Cyril and Methodius University, Skopje, Republic of North \\ Macedonia.
}

mejdins@gmail.com, bedrije_a@hotmail.com, vesname@pmf.ukim.mk

\section{Abstract}

We characterise the distributions generated by the boundary values of functions from Privalov spaces.

\section{Introduction}

We use the following notation and preliminaries. $U$ stands for the open unit disc in $C$ and $T$ is its boundary, i.e. $U=\{z \in C|| z \mid<1\}, T=\partial U$, and $\Pi^{+}$is the upper half plane, meaning $\Pi^{+}=\{z \in C \mid \operatorname{Imz}>0\}$. For a function $f$ holomorphic on a region $\Omega$ we right $f \epsilon H(\Omega)$. $L^{p}(\Omega)$ is the space of measurable functions on $\Omega$ such that $\int_{\Omega}|f(x)|^{p} d x<\infty ; L_{l o c}^{p}$ is the space of measurable functions on $\Omega$ such that for every compact set $K \subset \Omega$ the following holds $\int_{\mathrm{K}}|f(x)|^{p} d x<\infty$.

Privalov spaces on $U$ and $\Pi^{+}$and their properties: Privalov class, denoted with $N^{p}, 1<p<\infty$, consists of all functions $f \in H(\mathrm{U})$ such that

$$
\sup _{0 \leq r<1} \frac{1}{2 \pi} \int_{0}^{2 \pi}\left(\log ^{+} \mid f\left(r e^{i \theta} \mid\right)^{p} d \theta<\infty\right.
$$

Theorem. ([8]) The function $f$, holomorphic on $U$, belongs to $N^{p}$ if and only if for every $\varepsilon>0$ there exist $\delta>0$ such that for every measurable set $E \subset T$, satisfying $m(E)<\delta$ the following holds

$$
\int_{E}\left(\log ^{+}\left|f\left(r e^{i \theta}\right)\right|\right)^{p} d \theta<\varepsilon, \quad \text { for all } 0 \leq r<1 .
$$

Theorem. ([8]) The function $f$, holomorphic on $\mathrm{U}$, belongs to $N^{p}$ if and only if the subharmonic function $z \mapsto$ $\left(\log ^{+}|f(z)|\right)^{p}(z \epsilon U)$ has a harmonic majorant.

Every function in Nevalina class, $N(U)$, because of Fatou's lemma, has a nontangentional (radial) limit on $T$ almost everywere; every function in Privalov class, $N^{p}(U)$, has a nontangentional (radial) limit on $T$ almost everywere, in both cases we denote the boundary value with $f^{*}\left(e^{i \theta}\right)=\lim _{r \rightarrow 1} f\left(e^{i \theta}\right)$.

The class $N^{p}\left(\Pi^{+}\right), p>1$, is introduced analogously to $N^{p}(U)$, and is the set of all holomorphic functions on $\Pi^{+}$ satisfying

$$
\sup _{0<y<\infty} \int_{-\infty}^{\infty}\left(\log (1+|f(x+i y)|)^{p} d x<\infty\right.
$$

Every $f \in N^{p}\left(\Pi^{+}\right)$has a nontangentional limit $f^{*}(x)$ almost everywhere on the real axis.

Theorem. ([12]) The set $L$ is bounded in $N^{p}\left(\Pi^{+}\right)$if and only if 
i) There exist $C>0$ such that

for all $f \in L$.

$$
\int_{R}\left(\log \left(1+\left|f^{*}(x)\right|\right)^{p} d x<C\right.
$$

ii) For every $\varepsilon>0$, exist $\delta>0$ such that

$$
\int_{E}\left(\log \left(1+\left|f^{*}(x)\right|\right)^{p} d x<\varepsilon\right.
$$

for all $f \in L$, and every Lebesgue measurable $E \subset R$ satisfying $m(E)<\delta$.

Distributions: $C^{\infty}\left(R^{n}\right)$ denotes the set of all complex valued functions infinitely differentiable on $R^{n} ; C_{0}^{\infty}\left(R^{n}\right)$ is the subset of $C^{\infty}\left(R^{n}\right)$ which contains compactlly supported functions. Support of the function $f$ denoted with suppf is the cloasure of the set $\{x: f(x) \neq 0\}$ in $R^{n} . D=D\left(R^{n}\right)$ denotes the space $C_{0}^{\infty}\left(R^{n}\right)$ in which the convergence is defined in the following way: the sequence $\left\{\varphi_{\lambda}\right\}$, of functions $\varphi_{\lambda} \epsilon D$, converges to $\varphi \in D$ when $\lambda \rightarrow \lambda_{0}$ if and only if there exist compact subset of $R^{n}$ such that $\operatorname{supp} \varphi_{\lambda} \subseteq K$ for all $\lambda$, $\operatorname{supp} \varphi \subseteq K$, and for every n-tuple $\alpha$ of nonegative integers the sequence $\left\{D_{x}^{\alpha}\left(\varphi_{\lambda}(x)\right)\right\}$ converges to $\left\{D_{x}^{\alpha}(\varphi(x))\right\}$ uniformly on $\mathrm{K}$ when $\lambda \rightarrow \lambda_{0}$. With $D^{\prime}=D^{\prime}\left(R^{n}\right)$ is denoted the space of all continuous, linear functionals on $D$, where the continuity is in the sense: from $\varphi_{\lambda} \rightarrow \varphi$ in $D$ when $\lambda \rightarrow \lambda_{0}$ it follows that $\left\langle T, \varphi_{\lambda}\right\rangle \rightarrow\langle T, \varphi\rangle$ in $C$, when $\lambda \rightarrow \lambda_{0}$.

The space $D^{\prime}$ is called the space of distributions. We use the convention $\langle T, \varphi\rangle=T(\varphi)$ for the value of the functional Tacting on the function $\varphi$.

Let $\varphi \in D$ and $f(x) \in L_{l o c}^{1}\left(R^{n}\right)$. Then the functional $T_{f}$ on $D$ defined with

$$
\left\langle T_{f}, \varphi\right\rangle=\int_{R^{n}} f(t) \varphi(t) d t, \varphi \in D,
$$

is an element in $D^{\prime}$ and it is called the regular distribution generated by the function $f$.

\section{Main results}

Theorem. ([5]) Sufficient and necessary condition for the measurable function $\varphi\left(e^{i t}\right)$ defined on $T$ to coincide almost everywhere on $T$ with the boundary value $f^{*}\left(e^{i t}\right)$ of some function $f(z)$ in $N(U)$, is to exist a sequence of polynomials $\left\{P_{n}(z)\right\}$ such that:

i. $\left\{P_{n}\left(e^{i \theta}\right)\right\}$ converges to $\varphi\left(e^{i \theta}\right)$ almost everywhere on $\mathrm{T}$;

ii. $\varlimsup_{n \rightarrow \infty} \int_{0}^{2 \pi}\left(\log ^{+}\left|P_{n}\left(e^{i \theta}\right)\right|\right) d \theta<\infty$.

Theorem 1. Let $T_{f^{*}} \in D^{\prime}$ is generated by the boundary value $f^{*}(x)$ of a function $f(z)$ in $N^{p}\left(\Pi^{+}\right)$. There exist sequence of polynomials $\left\{P_{n}(z)\right\}, z \in \Pi^{+}$, and respectivelly $\left\{T_{n}\right\}, T_{n} \in D^{\prime}$, generated by the boundary values $P_{n}{ }^{*}(x)$ of the polynomials $P_{n}(z)$, i.e. $T_{n}=T_{P_{n}^{*}}$ such that:

i. $T_{n} \rightarrow T_{f^{*}}$ in $D^{\prime}$ when $n \rightarrow \infty$,

ii. $\varlimsup_{n \rightarrow \infty} \int_{-\infty}^{\infty}\left(\log \left(1+\left|P_{n}^{*}(x)\right|\right)\right)^{p}|\varphi(x)| d x<\infty$ for every $\varphi \in D$.

Proof. Let the assumptions of the theorem hold. Since $f \in N^{p}\left(\Pi^{+}\right)$, one has $f \in H\left(\Pi^{+}\right)$and there exist a constant $C>0$ such that

$\int_{-\infty}^{\infty} \log (1+|f(x+i y)|)^{p} d x \leq C$ for every $z=x+i y \in \Pi^{+}$.

Let $\left\{y_{n}\right\}$ be a sequence of positive real numbers satisfying $\lim _{n \rightarrow \infty} y_{n}=0$. We define a sequence of complex functions $\left\{F_{n}(z)\right\}$

$$
F_{n}(z)=f\left(z+i y_{n}\right)
$$

with

The functions $F_{n}(z)$ are holomorphic on $\Pi^{+} \cup R$. Margelijan theorem implies that for arbitrary compact subset $K$ of $\Pi^{+} \cup R$ with complement being connected, for the functions $F_{n}(z)$ there exist polynomials $P_{n}(z)$ such that $\left|F_{n}(z)-P_{n}(z)\right|<\varepsilon_{n}$, for all $z \in K$, where $\varepsilon_{n}>0$ and $\varepsilon_{n} \rightarrow 0$ when $n \rightarrow \infty$.

In what follows we prove i. and ii. 
i. Let

$\varphi \in D, \operatorname{supp} \varphi=K$.

Then

$$
\begin{gathered}
\left|\left\langle T_{n}, \varphi\right\rangle-\left\langle T_{f^{*}}, \varphi\right\rangle\right|=\left|\int_{-\infty}^{\infty} P_{n}^{*}(x) \varphi(x) d x-\int_{-\infty}^{\infty} f^{*}(x) \varphi(x) d x\right| \\
=\left|\int_{-\infty}^{\infty}\left[P_{n}^{*}(x)-f^{*}(x)\right] \varphi(x) d x\right|=\left|\int_{K}\left[P_{n}^{*}(x)-f^{*}(x)\right] \varphi(x) d x\right| \\
\leq M\left(\int_{K}\left[P_{n}^{*}(x)-f^{*}(x)\right] d x \leq M \varepsilon_{n}^{\prime} m(K) \rightarrow 0\right.
\end{gathered}
$$

when $n \rightarrow \infty$.

In the previous calculations we use the notation $m(K)$ for the Lebesgue measure of the set $K, M=$ $\max \{\varphi(x): x \in K\}$ and $\varepsilon_{n}^{\prime}=\varepsilon_{n}+\left[f^{*}(x)-F_{n}(x)\right]$. It is obvious that $\varepsilon_{n}^{\prime} \rightarrow 0$ when $n \rightarrow \infty$. The Later calculation implies that $\left\langle T_{n}, \varphi\right\rangle \rightarrow\left\langle T_{f^{*}}, \varphi\right\rangle$ when $n \rightarrow \infty$ for every, but fixed, $\varphi \in D$, meaning $T_{n} \rightarrow T_{f^{*}}$ weakly in $D^{\prime}$. To prove the convergence in the strong topology it sufficies to prove the same convergence for $\varphi \in B$ for an arbitrary bounded set in $D$. Choose $B \subset D$, arbitrary bounded set. The condition of boundnes implies that there exists a compact set $K$ such that $\operatorname{supp} \varphi \in K,\|\varphi\|_{D(K)}<M$, for every $\varphi \in B$. Note that the calculations at the beginning of the paragraph hold for every $\varphi \in B$ and the new compact set chosen for the boundness condition. Hence, $T_{n} \rightarrow T_{f^{*}}$ in $D^{\prime}$.

(ii)

$$
\begin{gathered}
\int_{-\infty}^{\infty}\left(\log \left(1+\left|P_{n}^{*}(x)\right|\right)^{p}|\varphi(x)| d x\right. \\
=\int_{K}\left(\log \left(1+\left|P_{n}^{*}(x)+F_{n}(x)-F_{n}(x)\right|\right)^{p}|\varphi(x)| d x\right. \\
\leq \int_{K}\left(\log \left(1+\left|P_{n}^{*}(x)-F_{n}(x)\right|+\left|F_{n}(x)\right|\right)\right)^{p}|\varphi(x)| d x \\
\leq \int_{K}\left(\log \left(1+\left|F_{n}(x)\right|+\left|P_{n}^{*}(x)-F_{n}(x)\right|\right)\right)^{p}|\varphi(x)| d x \\
\leq M 2^{p-1} \int_{K}\left(\log \left(1+\left|F_{n}(x)\right|\right)^{p} d x+M 2^{p-1} \int_{K}\left|P_{n}^{*}(x)-F_{n}(x)\right|\right)^{p} d x \\
\leq M C+M \varepsilon_{n}^{p} m(K) .
\end{gathered}
$$

Because $\varepsilon_{n} \rightarrow 0, n \rightarrow \infty$ we get $\int_{R}\left(\log \left(1+\left|\mathrm{P}_{\mathrm{n}}^{*}(\mathrm{x})\right|\right)^{\mathrm{p}}|\varphi(x)| d x<C^{\prime}\right.$ meaning $\varlimsup_{n \rightarrow \infty} \int_{-\infty}^{\infty}\left(\log \left(1+\left|P_{n}^{*}(x)\right|\right)^{n}|\varphi(x)| d x<\infty\right.$, for all $\varphi \in D$.

In the proof of ii. We use the inequalities $|a+b| \leq|a|+|b|, \log (1+a+b) \leq \log (1+a)+b$, for $a, b>0$ and $(a+b)^{p} \leq 2^{p-1}\left(a^{p}+b^{p}\right)$, for $p \geq 1$.

Theorem 2. Let $\varphi_{0}$ be a localy integrable function and $T_{\varphi_{0}} \in D^{\prime}$ is generated by the function $\varphi_{0}$. Let there exist sequence of polynomials $P_{n}(z)$ satisfying the conditions:

i. The sequence of distributions generated by the boundary values $P_{n}^{*}(x)$ of $P_{n}(z)$ converges to $T_{\varphi_{0}}$ in $D$ when $n \rightarrow \infty$;

ii. $\quad \varlimsup_{n \rightarrow \infty}^{\infty} \int_{-\infty}^{\infty}\left(\log \left(1+P_{n}(x+i y)\right)^{p}|\varphi(x)| d x<C<\infty, \forall z=x+i y \in \Pi^{+}, \varphi \in D\right.$.

There exists a function $f \in H\left(\Pi^{+}\right)$such that 
for every compact $K \subset R$, and

$$
\int_{K}\left(\log (1+|f(x+i y)|)^{p} d x<C<\infty, \forall z=x+i y \in \Pi^{+},\right.
$$

$$
\lim _{y \rightarrow 0^{+}} \int_{-\infty}^{\infty} f(x+i y) \varphi(x) d x=\left\langle T_{\varphi_{0}}, \varphi\right\rangle
$$

Proof. Let the assumptions of the theorem are fulfilled. In [3] it is proven that from i., i.e.

$\lim _{n \rightarrow \infty} \int_{R} P_{n}^{*}(x) \varphi(x) d x=\int_{R} \varphi_{0}(x) \varphi(x) d x, \varphi \in D$,

implies the existence of $f \in H\left(\Pi^{+}\right)$such that the sequence of polynomials converges to $f$, uniformly on arbitrary compact subsets of $\Pi^{+}$when $n \rightarrow \infty$.

Firstly we will prove that this function $f$ is holomorphic and satisfies the condition

$$
\int_{K} \log (1+|f(x+i y)|)^{p} d x \leq C
$$

for all $z=x+i y \in \Pi^{+}$and arbitrary compact set $K \subset R$.

Indeed, we use the condition ii., i.e.

$\varlimsup_{n \rightarrow \infty} \int_{-\infty}^{\infty}\left(\log \left(1+\left|P_{n}(x+i y)\right|\right)^{p}|\varphi(x)| d x<C<\infty, \forall z=x+i y \in \Pi^{+}, \varphi \in D\right.$.

Let $K$ be compact set. There exists $\varphi(x) \epsilon C_{0}^{\infty}\left(R^{n}\right), \varphi(x)=1, \forall x \in K$. To obtain the last statement, it is enough to take characteristic function of the set $K$ and to regularize it. Substitution of such $\varphi$ in to ii., implies that for every $n \in N$,

Now,

$$
\int_{K}\left(\log \left(1+\left|P_{n}(x+i y)\right|\right)\right)^{p} d x<C<\infty, \forall z=x+i y \in \Pi^{+} .
$$

$$
\begin{gathered}
\int_{K} \log (1+|f(x+i y)|)^{p} d x=\int_{K} \lim _{n \rightarrow \infty}\left(\log \left(1+\left|P_{n}(x+i y)\right|\right)^{p}\right. \\
\leq \varlimsup_{n \rightarrow \infty} \int_{-\infty}^{\infty}\left(\log \left(1+\left|P_{n}(x+i y)\right|\right)^{p} d x<C<\infty\right.
\end{gathered}
$$

i.e.

$\int_{K} \log (1+|f(x+i y)|)^{p} d x \leq C<\infty$ for arbitrary compact set $K \subset R$ and every $z=x+i y \in \Pi^{+}$.

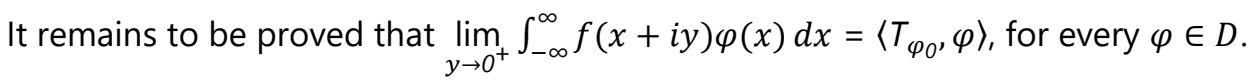

Let $\varphi \in D$ and $\operatorname{supp} \varphi=K \subset R$. Then

$$
\begin{gathered}
\lim _{y \rightarrow 0^{+}} \int_{R}\left(f(x+i y) \varphi(x) d x=\lim _{y \rightarrow 0^{+}} \int_{R} \lim _{n \rightarrow \infty}\left(P_{n}(x+i y) \varphi(x) d x=\right.\right. \\
=\lim _{y \rightarrow 0^{+}} \lim _{n \rightarrow \infty} \int_{K}\left(P_{n}(x+i y) \varphi(x) d x=\lim _{n \rightarrow \infty} \lim _{y \rightarrow 0^{+}} \int_{K}\left(P_{n}(x+i y) \varphi(x) d x=\right.\right. \\
=\lim _{n \rightarrow \infty} \int_{K} P_{n}^{*}(x+i y) \varphi(x) d x=\int_{R} \varphi_{0}(x) \varphi(x) d x=\left\langle T_{\varphi_{0^{\prime}}} \varphi\right\rangle,
\end{gathered}
$$

for every $\varphi \in D$.

The previous equalities are obvious, exept the following

$$
\lim _{y \rightarrow 0^{+}} \lim _{n \rightarrow \infty} \int_{K} P_{n}(x+i y) \varphi(x) d x=\lim _{n \rightarrow \infty} \lim _{y \rightarrow 0^{+}} \int_{K} P_{n}(x+i y) \varphi(x) d x
$$

for $z=x+i y \in \Pi^{+}$. 
We will prove $(*)$.

To do that we consider the sequence of functions $\left\{g_{n}(y)\right\}$ defined by

$$
g_{n}(y)=\int_{K}\left(P_{n}(x+i y) \varphi(x) d x, x+i y \in K_{1} .\right.
$$

for $K_{1}$ compact subset of $\Pi^{+}$such that $z \in K_{1}$ for $\operatorname{Re}(z) \in K$. Because $\left\{P_{n}(x+i y)\right\}$ converges to $(x+i y)$ uniformly on $K_{1}$, when $n \rightarrow \infty$, one obtains that for fixed $y$

$$
\lim _{n \rightarrow \infty} g_{n}(y)=\int_{K}\left(P_{n}(x+i y) \varphi(x) d x=\int_{K}(f(x+i y) \varphi(x) d x=g(y),\right.
$$

i.e. the sequence $\left\{g_{n}(y)\right\}$ converges to $g(y)$ when $n \rightarrow \infty$. We will prove that this convergence is uniform on $\operatorname{Im} K_{1}$, which will imply the statement. Indeed,

$$
\begin{aligned}
0 \leq \sup _{y} \mid g_{n}(x+i y) & -g(x+i y)\left|=\sup _{y}\right| \int_{K} \mathrm{P}_{\mathrm{n}}(\mathrm{x}+\mathrm{iy}) \varphi(\mathrm{x}) \mathrm{dx}-\int_{K} f(x+i y) \varphi(x) d x \mid \\
& =\sup _{y}\left|\int_{K}\left[P_{n}(x+i y)-f(x+i y)\right] \varphi(x) d x\right| \\
& \leq \sup _{y} \int_{K} \mid\left(P_{n}(x+i y)-(f(x+i y)|| \varphi(x) \mid d x\right. \\
& \leq M \sup _{y} \int_{K}\left|P_{n}(x+i y)-f(x+i y)\right| d x .
\end{aligned}
$$

Since

$$
P_{n}(x+i y) \rightarrow f(x+i y)
$$

uniformly

on

$K_{1}$

it

follows

that

converges to 0 uniformly on $\operatorname{Im}\left(K_{7}\right)$ meaning

$$
\int_{K}\left|P_{n}(x+i y)-f(x+i y)\right| d x
$$

$$
\limsup _{n \rightarrow \infty} \int_{K} \mid\left(P_{n}(x+i y)-f(x+i y) \mid d x=0 .\right.
$$

Finally, $\lim _{n \rightarrow \infty} \sup _{y}\left|g_{n}(x+i y)-g(x+i y)\right|=0$.

\section{Conclusion}

We obtain necessary and sufficient condition for a distribution generated from an element of the Privalov class to be boundary value of analytic functions on upper half space. The boundary values are taken in the distributional sense.

\section{References}

1. Ansari,A.H., Liu,X. and Mishra ,V.N.(2017) On Mittag-Leffler function and beyond. Nonlinear Science Letters A, Vol. 8, No. 2, pp. 187-199.

2. Bremermann, G. Raspredelenija, kompleksnije permenenije i preobrazovanija Fourie.1968. Moskva.Mir

3. Duren, P. L., Theory of $H^{p}$ Spaces. 1970. New York. Acad. Press.

4. lida, Y. (2017). Bounded Subsets of Smirnov and Privalov Classes on the Upper Half Plane. Hindawi International Journal of Analysis. Article ID 9134768, 4 pages. doi.org/10.1155/2017/9134768.

5. Manova, E. V. (2002). Bounded subsets of distributions in D' generated with boundary values of functions of the space $\mathrm{H}^{\mathrm{p}}, 1 \leq \mathrm{p}<\infty$. Godisenj zbornik na Insitut za matematika, Annuaire, ISSN 0351724, pp. 31-40. 
6. Manova, E. V.(2001). Distributions generated by boundary values of functions of the Nevanlina class N. Matematichki vesnik, Knjiga 54, Sveska 3-4, Beograd, Srbija i Crna Gora, YU ISSN 0025-5165, pp.133-138.

7. Meštrović,R. and Pavićević, Z.(2017). A short survey of some topologies on Privalov spaces on the unit disk", Math. Montisnigri 40, pp. 5-13.

8. Meštrović,R. and Pavićević, Z.(2014). A topological property of Privalov spaces on the unit disk. Math. Montisnigri 31, pp. 1-11.

9. Meštrović,R. and Sušić, Z.(2013). Interpolation in the spaces $\mathrm{Np}(1<\mathrm{p}<\infty)$. Filomat 27, pp. 293301.

10. Meštrović, R. and Pavićević, Z.(2015). On some metric topologies on Privalov spceson the unit disc. Math. FA.

11. Privalov, I. I.,1941. Granicnije svojstva odnoznacnih analitickih funkcijii. Moskva,Nauka.

12. Reckovski, V., Manova, E.V. and Reckoski N.(2015). Convergence of some special harmonic functions. Proceedings of the $V$ Congress of the Mathematicians of Macedonia, Vol. 2 CMM, Macedonia, p.p. 53-57.

13. Reckovski, V., Manova,E.V. Bedjeti,B.and Iseni,E.(2019). For some boundary value problems in distributions. Journal of Advances in Mathematics, Volume 16, Khalsa Publications, ISSN: 2347-1921, pp. 8331-8339.

\section{Conflicts of Interest}

The authors don't have competing for any interests

\section{Acknowledgments}

The authors are grateful to the referees for their valuable suggestions. 